\title{
MICROBIOLOGICAL PROFILE OF NEONATAL SEPTICAEMIA ALONG WITH AN OVERVIEW OF THE CLINICAL SCENARIO
}

Vaibhav Vitthalrao Rajhans ${ }^{1}$, Sharmila Sanjay Raut², Sanjaykumar Rajaram More ${ }^{3}$, Vimal Shriram Rathod ${ }^{4}$, Vivek Madhukarrao Gujar ${ }^{5}$

${ }_{1}^{1}$ Assistant Professor, Department of Microbiology, RMC, Loni.

2 Professor and HOD, Department of Microbiology, IGMC, Nagpur.

3 Professor and HOD, Department of Microbiology, SRTRGMC, Ambajogai.

${ }^{4}$ Associate Professor, Department of Microbiology, SCGMC, Nanded.

${ }_{5}^{5}$ Associate Professor, Department of Microbiology, VNGMC, Yavatmal.

\begin{tabular}{l}
\hline ABSTRACT \\
\hline BACKGROUND \\
Neonatal sepsis takes a big toll of neonatal deaths in developing countries. It is estimated that up to $20 \%$ of the neonates develop \\
sepsis and approximately $1 \%$ die of sepsis related causes. In India, sepsis is one of the commonest causes of neonatal mortality \\
contributing to $19 \%$ of all neonatal deaths. \\
The present study was undertaken to study aerobic bacterial profile of neonatal septicaemia by automated blood culture systems.
\end{tabular}

\section{MATERIALS AND METHODS}

This observational study was conducted in the Microbiology Laboratory of a tertiary care centre over a period of one and a half years [January 2013 to June 2014]. Blood samples of neonates of both sexes were collected and processed. Detailed history recording and clinical examination was conducted on each patient and findings were recorded.

\section{RESULTS}

Total 157 blood samples, 99 [63.05\%] from male and 58 [36.94\%] from female patients were processed. Out of 157 blood samples, 70 i.e. 44.58\% were flagged as positive by BacT/ ALERT 3D 60 system and 87 [55.41\%] were negative. Within the neonatal age group, 52 [74.28\%] neonates with positive blood cultures were of the age less than one week. Out of 70 culture positive cases 64 [91.42\%] deliveries were conducted in the hospitals, rest 6 [8.57\%] were home-based deliveries. Out of 70, 12 [17.14\%] neonates were having history of febrile illness in the mother during gestation. And 17 [24.28\%] neonates were found to have history of Prolonged Rupture of Membranes [PROM] during delivery. 43 [61.42\%] of the 70 positive cases had not attained their gestational maturity. Out of 70 blood culture positive neonates, 44 [62.85\%] were having early-onset sepsis. Rest 26 [37.14\%] were suffering from late-onset sepsis. All 70 blood cultures were monobacterial. Out of 70 isolates, 35 [50\%] isolates were Gram-positive cocci and 35 [50\%] isolates were Gram-negative bacilli. Least susceptibility of E. coli isolates was observed to Cefepime [18.75\%] and Ampicillin [12.50\%].

\section{CONCLUSION}

Culture positivity was found more in male neonates as compared to females. The male-to-female ratio being 1.69: 1. Highest blood culture positivity, i.e. $74.28 \%$ was found in neonates of age less than one week. $17.14 \%$ out of 70 babies with positive culture were having history of maternal pyrexia during gestation. $24.28 \%$ cases were found to have prolonged rupture of membranes during deliveries. $61.42 \%$ cases were preterm deliveries. $60 \%$ babies were having low birth weight. $62.85 \%$ cases were having early-onset sepsis and $37.14 \%$ were of late-onset sepsis.

\section{KEYWORDS}

Neonatal Sepsis, Blood Culture, Automated Blood Culture, BacT/ ALERT, MiniAPI

HOW TO CITE THIS ARTICLE: Rajhans VV, Raut SS, More SR, et al. Microbiological profile of neonatal septicaemia along with an overview of the clinical scenario. J. Evolution Med. Dent. Sci. 2018;7(04):411-418, DOI: 10.14260/jemds/2018/92

\section{BACKGROUND}

Neonatal sepsis is responsible for $30 \%-50 \%$ of the total neonatal deaths in developing countries. It is estimated that up to $20 \%$ of the neonates develop sepsis and approximately $1 \%$ die of sepsis related causes. The incidence of neonatal sepsis according to the data from National Neonatal Perinatal

'Financial or Other Competing Interest': None.

Submission 06-11-2017, Peer Review 22-12-2017,

Acceptance 28-12-2017, Published 22-01-2018.

Corresponding Author:

Vaibhav Vitthalrao Rajhans,

Assistant Professor

Department of Microbiology,

RMC, Loni, Tal. Rahata Dist.,

Ahmednagar, Maharashtra.

E-mail: vaibhavrajhans@gmail.com

DOI: $10.14260 /$ jemds $/ 2018 / 92$

\section{(c) (1) $(9)$}

Database is 30 per 1000 live births. This database comprising of various tertiary care neonatal units across India found sepsis to be one of the commonest causes of neonatal mortality contributing to $19 \%$ of all neonatal deaths. ${ }^{1}$

Globally, an estimated 4 million babies die in the first 4 weeks of life (the neonatal period) every year and half of them die in their first 24 hours. $^{2}$ It accounts for $40 \%$ of under 5 mortality. $98 \%$ of these deaths occur in developing countries. ${ }^{3}$

In India, the Sample Registration System [SRS] estimates of neonatal mortality for the year 2010 of about 25 per 1000 live births in early neonatal period [0 - 7 days] with 28 for rural areas and 15 for urban areas. ${ }^{3}$

Considering these grave consequences, there is an urgent need to know whether the baby has sepsis to institute treatment as quickly as possible. The gold standard for 
diagnosis of septicaemia is the isolation of bacterial agents from blood culture. ${ }^{1}$

Hence, the present study was undertaken to study aerobic bacterial profile of neonatal septicaemia by automated blood culture systems using BacT/ALERT 3D 60 blood culture system and miniAPI bacterial identification and antibiotic susceptibility testing system, both by Biomerieux.

\section{MATERIALS AND METHODS}

This observational study was conducted in the Microbiology Laboratory of this tertiary care centre over a period of one and a half years [January 2013 to June 2014]. Ethical clearance was obtained from the Institutional Ethical Clearance Committee.

Blood samples of all patients in the neonatal age group [0 - 28 days of age] of both sexes who got admitted in the ward at this tertiary care centre during the study period with clinical suspicion of septicaemia were collected and processed. Detailed history recording, and clinical examination was conducted on each patient and findings were recorded.

\section{Sample Collection}

1 - $2 \mathrm{~mL}$ of blood sample from each neonate clinically suspected to be having septicaemia was collected using all standard aseptic precautions and inoculated in special paediatric blood culture media bottle- The BacT/ Alert PF bottle containing $20 \mathrm{~mL}$ broth. ${ }^{4-7}$

The inoculated bottles were loaded into the BacT/ Alert 3D/60 system incubator and incubated for a maximum period of 5 days. ${ }^{8,9}$

\section{Processing of Positive Blood Culture Bottles}

Blood culture bottles flagged as positive by this instrument were unloaded from the system and processed further according to Manufacturer's instructions using miniAPI Identification [ID] and Antibiotic susceptibility [ATB] strips on miniAPI system. The strips that were used in this study were as follows-

ID strips- rapid ID 32 E, ID 32 GN, ID 32 STAPH and rapid ID 32 STREP.

ATB strips- rapid ATB E 4, ATB PSE 5, ATB STAPH 5, ATB STREP 5 and ATB ENTEROC 5.

\section{RESULTS}

Total 157 blood samples, 99 [63.05\%] from male and 58 [36.94\%] from female patients were processed.

\section{Blood Culture Report}

\begin{tabular}{|c|c|c|}
\hline Result by BacT/ Alert & No. of Samples & Percentage \\
\hline Positive & 70 & $44.58 \%$ \\
\hline Negative & 87 & $55.41 \%$ \\
\hline Total & 157 & $100 \%$ \\
\hline $\begin{array}{r}\text { Table/Figure No. 1. Blood Culture Report by BacT/ } \\
\text { Alert 3D 60 Automated System (n= 157) }\end{array}$ \\
\hline
\end{tabular}

Out of 157 blood samples, 70 i.e. $44.58 \%$ were flagged as positive by BacT/ Alert 3D 60 system and 87 [55.41\%] were negative (Table/ Figure No. 1).
Sex-Wise distribution of Culture Positive Cases

In sex-wise distribution of culture positive cases positivity was found more in male patients, which was 44 [62.85\%] out of 70. Female patients comprised of 26 [37.14\%] of the positive samples. The male-to-female ratio observed was 1.69: 1 (Table/ Figure No. 2).

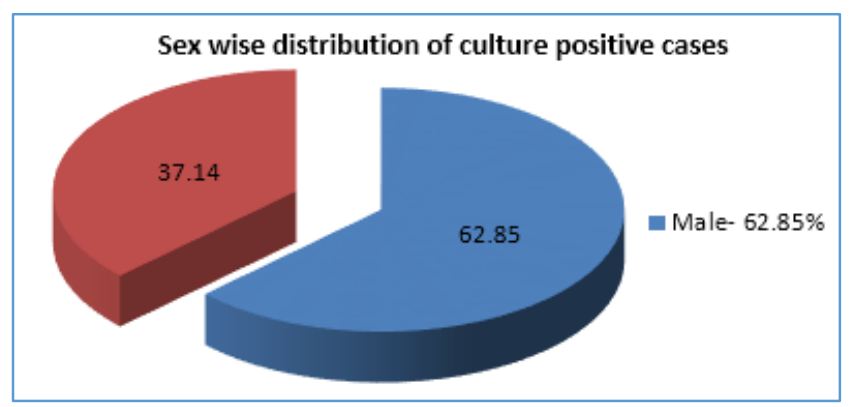

Table/Figure No. 2. Sex Wise distribution of Culture Positive Cases

Age Wise distribution of Culture Positive Cases

Within the neonatal age group, 52 [74.28\%] neonates with positive blood cultures were of the age less than one week. Neonates between the age one week to two weeks comprised of 14 [20\%] of the 70 positive samples. 4 [5.71\%] neonates were more than two weeks old (Table/ Figure No. 3).

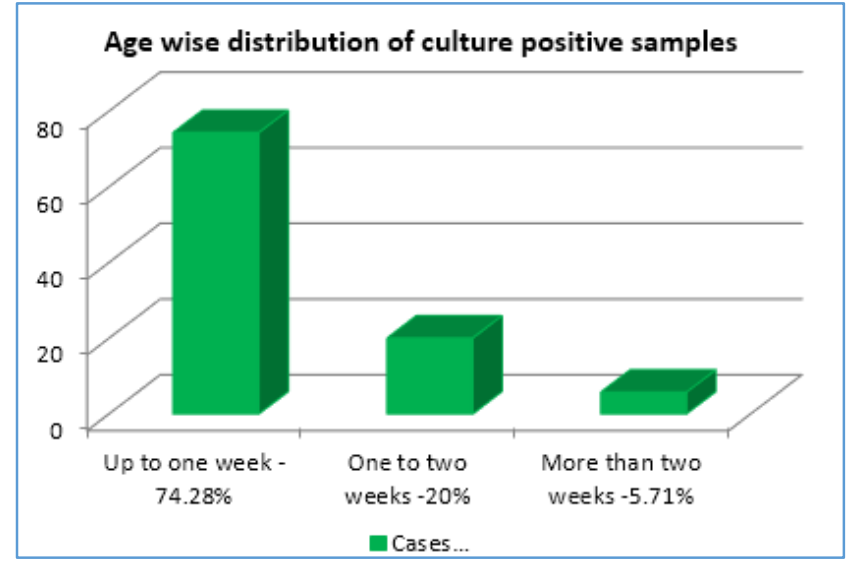

Table/ Figure No. 3. Age Wise distribution of Culture Positive Cases

Distribution of Culture Positive Cases according to Place and Mode of Delivery

\begin{tabular}{|c|c|c|c|c|c|}
\hline \multirow{2}{*}{$\begin{array}{l}\text { Place of } \\
\text { Delivery }\end{array}$} & \multicolumn{3}{|c|}{ Hospital } & \multirow{2}{*}{$\begin{array}{l}\text { Home } \\
\text { Based }\end{array}$} & \multirow[b]{2}{*}{ Total } \\
\hline & NVD & LSCS & $\begin{array}{c}\text { Assisted } \\
\text { Deliveries }\end{array}$ & & \\
\hline $\begin{array}{c}\text { Number of } \\
\text { cases }\end{array}$ & 47 & 13 & 4 & 6 & 70 \\
\hline$\%$ & 67.14 & 18.57 & 5.71 & 8.57 & 100 \\
\hline
\end{tabular}

Out of 70 culture positive cases 64 [91.42\%] deliveries were conducted in the hospitals, rest 6 [8.57\%] were homebased deliveries. Out of 64 hospital-based deliveries 47 [67.14\%] were Normal Vaginal Deliveries [NVD], Lower Segment Caesarean Section [LSCS] was done in 13 [18.57\%] cases and 4 [5.71\%] were assisted deliveries requiring instrumentation (Table/ Figure No. 4). 
Distribution of Culture Positive Cases according to associated Maternal Risk Factors

Out of 70, 12 [17.14\%] neonates were having history of febrile illness in the mother during gestation and 17 [24.28\%] neonates were found to have history of Prolonged Rupture of Membranes [PROM] during delivery.

Distribution of Culture Positive Cases according to the Gestational Age

Prematurity is one of the most important predisposing factors for neonatal septicaemia. In this study, those neonates who were confirmed culture positive showed distribution consistent with this. 43 [61.42\%] of the 70 positive cases have not attained their gestational maturity. However, as gestational age is not the sole determining phenomenon for this condition, neonatal septicaemia was evident in 27 [38.57\%] patients who had completed their term before birth (Table/ Figure No. 5).



Table/Figure No. 5. Distribution of Culture Positive Cases according to Gestational Age at the Time of Birth

Distribution of Culture Positive Cases according to the Birth Weight

\begin{tabular}{|c|c|c|}
\hline $\begin{array}{c}\text { Birth Weight of the } \\
\text { Newborn }\end{array}$ & $\begin{array}{c}\text { Number of Culture } \\
\text { Positive Cases (n= 70) }\end{array}$ & $\mathbf{\%}$ \\
\hline Less than 1500 gm & 07 & 10 \\
\hline 1501-2500 gm & 35 & 50 \\
\hline More than 2500 gm & 28 & 40 \\
\hline Total & 70 & 100 \\
\hline Table/Figure No. 6. Birth Weight Wise distribution of \\
Culture Positive Cases \\
\hline
\end{tabular}

Out of 70 blood culture positive neonates, 28 [40\%] were having birth weight more than 2500 gm. 42 [60\%] neonates were having birth weight less than $2500 \mathrm{gm}$. Out of these 42 , 35 [50\%] neonates were having birth weight of 1500 - 2500 gm, 7 [10\%] babies were having birth weight less than 1500 gm (Table/ Figure No. 6).

Distribution of Culture Positive Cases according to the Onset of Sepsis

\begin{tabular}{|c|c|c|}
\hline Type of Sepsis & No. of Cases $(n=70)$ & $\%$ \\
\hline Early-onset sepsis [EOS] & 44 & 62.85 \\
\hline Late-onset sepsis [LOS] & 26 & 37.14 \\
\hline Total & 70 & 100.00 \\
\hline \multicolumn{3}{|c|}{$\begin{array}{c}\text { Table/Figure No. 7. Distribution of Culture Positive Cases } \\
\text { according to the Onset of Sepsis }\end{array}$} \\
\hline
\end{tabular}

Out of 70 blood culture positive neonates, 44 [62.85\%] were having early-onset sepsis. Rest 26 [37.14\%] were suffering from late-onset sepsis (Table/ Figure No. 7).

\section{Bacteriology}

Distribution of culture positive cases according to Gram reaction of the isolate. All 70 blood cultures were monobacterial. Out of 70 isolates, 35 [50\%] isolates were Gram-positive cocci and 35 [50\%] isolates were Gramnegative bacilli. Hence, Gram-positive and negative organisms contributed in equal proportion to the microbiological aetiology of neonatal septicaemia.

\section{Organism Wise distribution of Culture Positive Cases}

Of the total 70 isolates E. coli was the most common, i.e. 16 [22.85\%] followed by Coagulase-negative Staphylococcus [CoNS] isolated in 15 [21.42\%] followed by Staphylococcus aureus isolated in 14 [20\%], Klebsiella pneumonia 10 [14.28\%], Pseudomonas aeruginosa 6 [8.57\%], Enterococcus faecalis 3 [4.28\%] and Streptococcus pyogenes 2 [2.85\%]. While Citrobacter koseri, Enterobacter cloacae, Burkholderia cepacia and Enterococcus faecium were isolated from 1 [1.42\%] sample each (Table/ Figure No. 8).

\section{Bacterial isolates- cases in \%}



Table/ Figure No. 8. Organism Wise distribution of Isolates from Positive Blood Samples

\section{Antibiotic Susceptibility Patterns}

ATB strip used for Staphylococci contained 16 antibiotics. Among Coagulase negative staphylococci [CoNS], maximum susceptibility was found to the Quinupristin-Dalfopristin [100\%] and Vancomycin [100\%] followed by Minocycline [93.33\%] and Levofloxacin [86.66\%]. Least susceptibility by CoNS was shown to Erythromycin [40\%] and to Penicillin [13.33\%].

Staphylococcus aureus showed maximum susceptibility to the Quinupristin-Dalfopristin [100\%] and Vancomycin [100\%] followed by Nitrofurantoin [92.85\%] and Tetracycline [85.71\%]. Cotrimoxazole and Penicillin were the drugs to which Staphylococcus aureus isolates were least susceptible, $42.85 \%$ and $21.42 \%$, respectively.

ATB strips used for Enterobacteriaceae contained 30 different antibiotics.

E. coli isolates were most susceptible to Piperacillin + Tazobactam [93.75\%] and Nitrofurantoin [93.75\%] followed by Ticarcillin + Clavulanic acid [87.5\%]. Least susceptibility of E. coli isolates was observed to Cefepime [18.75\%] and Ampicillin [12.50\%]. 
Among Klebsiella pneumoniae isolates, maximum susceptibility was shown to Piperacillin + Tazobactam [90\%] and Piperacillin [80\%] followed by Ciprofloxacin [70\%] and Ceftriaxone [70\%]. Least susceptibility was shown to Ampicillin [20\%], cefoxitin [20\%] and Cefazolin [10\%].

Citrobacter koseri was observed to be susceptible to all antibiotics except Ampicillin, Cefepime, Ticarcillin, Amikacin, Chloramphenicol, Cephalothin, Cotrimoxazole, Cefazolin, Cefoxitin, Ofloxacin, Cefuroxime, Ciprofloxacin, Levofloxacin, Fosfomycin, Ceftazidime and Nitrofurantoin.

Enterobacter cloacae was observed to be susceptible to all antibiotics except Ampicillin, Cefepime, Ticarcillin, Chloramphenicol, Cephalothin, Cotrimoxazole, Cefazolin, Cefoxitin, Cefuroxime, Ciprofloxacin, Levofloxacin and Ceftazidime.

ATB strips used for Pseudomonas contained 15 different antibiotics.

Pseudomonas aeruginosa were most susceptible to Imipenem [100\%], Meropenem [100\%] and Piperacillin + Tazobactam [83.33\%] followed by Amikacin [66.66\%] and Gentamicin [66.66\%]. These isolates were least susceptible to Cefepime [33.33\%] and Ticarcillin [16.66\%].

The single Burkholderia cepacia isolate was sensitive to Imipenem, Meropenem, Piperacillin + Tazobactam, Amikacin, Gentamicin, Tobramycin, Ciprofloxacin, Piperacillin, Ampicillin + Sulbactam and Ticarcillin + Clavulanic acid. But it was found resistant to Ticarcillin, Cefepime, Ceftazidime, Cotrimoxazole and Colistin.

The ATB strips for Streptococcus pyogenes contained 10 antibiotics.

Both Streptococcus pyogenes isolates were susceptible to Cefotaxime, Quinupristin-Dalfopristin, Clindamycin, Vancomycin and both were resistant to Penicillin.

The strips used for Enterococcus species contained 14 antibiotics.

All Enterococcus faecalis isolates were susceptible to Streptomycin, Vancomycin, Teicoplanin and QuinupristinDalfopristin. All E. faecalis were resistant to Penicillin. Enterococcus faecium isolate was susceptible to all antibiotics except Penicillin and Nitrofurantoin.

\section{Survival Rate among the Culture Positive Cases}

Among the 70 neonates who were diagnosed to be having septicaemia by positive blood cultures, 58 [82.85\%] improved and were discharged from the wards. 12 [17.14\%] neonates succumbed to their illness.

\section{DISCUSSION}

\section{Blood Culture Positivity}

Ghanshyam et al [2002] in their study observed $42.00 \%$ positivity in blood cultures. ${ }^{10}$ Ananthakrishnan et al ${ }^{11}$ [2009] in their study concluded that $40.6 \%$ samples were positive in blood culture. According to Gandhi S et al [2013], out of 238 samples studied blood culture was positive in 76 cases (32\%). ${ }^{1}$ Chelliah A et al [2014] mentioned that there were 182 clinically suspected cases of neonatal sepsis. A culture positivity rate of 110 (60.4\%) was observed.12 Basu R et al [2014] observed blood culture positivity in 119 [39.66\%] among 300 subjects of neonatal age group. ${ }^{13}$ Roy I et al [2002] observed growth of bacteria in 346 (47.5\%) blood samples out of 728.14 Mahapatra A et al [2002] observed that out of the 120 blood cultures, 48 (40\%) were bacteriologically positive. ${ }^{15}$

It shows that the culture positivity for aerobic organisms in neonates varies from $20 \%$ to $60 \%$. Only aerobic bacteria were studied in the present study.

Blood culture positivity in this study was $44.58 \%$ which is comparable with study by Ghanshyam et al, Roy et al and Mahapatra et al.10,14,15

\section{Sex Wise distribution of Culture Positive Cases}

In the study by Jyothi $\mathrm{P}$ et al, among the culture positive cases there were 86 (65.5\%) male and 45 (34.5\%) female neonates with male-to-female ratio of 1.9: 1.16 Haseeb M et al [2014] described that analysis with respect to sex of the baby showed that 65 cases (61.9\%) were males and 40 cases $(38.09 \%)$ were females with a male: female ratio of 1.62: 1.17 Gheibi et al [2008] observed the male-to-female ratio as $1.67: 1$ in a study of 227 cases. ${ }^{18}$

Our observation is similar to that of Khatua et al and Gheibi et al.19,18 Khatua et al $^{19}$ postulated that the factors regulating the synthesis of gamma globulins are probably situated on the $\mathrm{X}$ chromosome. Presence of one $\mathrm{X}$ chromosome in the male infant thus confers less immunological protection compared to female counterpart. Singh $^{20}$ stated that male infants are around 4 times at increased risk to develop sepsis compared to females. Term male infants have an approximately two-fold higher incidence of septicaemia than term females. ${ }^{21}$

\section{Age Wise distribution of Culture Positive Cases}

Within the neonatal age group, 52 [74.28\%] neonates with positive blood cultures were of age less than one week Neonates between the age of one week to two weeks comprised 14 [20\%] of the 70 positive samples. 4 [5.71\%] neonates were more than two weeks old.

Patel U et al [2014] $]^{22}$ observed in their study that $52.8 \%$ of neonates with clinical suspicion of sepsis belonged to age group of less than 1 week. $45.2 \%$ babies were of age more than 1 week.

\section{Distribution of Culture Positive Cases according to Place and Mode of Delivery}

In the present study, out of 70 culture positive cases 64 [91.42\%] deliveries were conducted in the hospitals, rest 6 [8.57\%] were home-based deliveries. Out of 64 hospitalbased deliveries, 47 [67.14\%] were Normal Vaginal Deliveries [NVD]. Lower Segment Caesarean Section [LSCS] was done in 13 [18.57\%] cases and 4 [5.71\%] were assisted deliveries requiring instrumentation.

Kuruvilla et $\mathrm{al}^{23}$ observed normal delivery in $48.51 \%$ cases. LSCS was performed in $38.06 \%$ cases, whereas instrumentation in $13.43 \%$ cases. Ahmed et al ${ }^{24}$ reported that more than half of the culture positive neonates were delivered at home $(60 \%)$ and 7 [23\%] culture positive babies were delivered by caesarean section. In the study of Knudsen et $\mathrm{al}^{25}$ the majority of the study population was poor and delivered at home, largely in the hands of untrained birth attendants. Home deliveries are significantly related to birth asphyxia, which was highly prevalent in population and which in turn is associated with an increased risk of serious neonatal infection. 


\section{Distribution of Culture Positive Cases according to associated Risk Factors}

Infection of the mother at the time of birth, particularly genital infection, is the principal pathway of maternal transmission ${ }^{26,27}$ and can play an important role in the development of infection in the neonate. Transplacental haematogenous infection during or shortly before delivery (including the period of separation of the placenta) is possible, although it seems more likely that the infant is infected during passage through the birth canal.

Soman et $\mathrm{al}^{28}$ found the maternal fever as the predisposing factor of neonatal septicaemia in $18.6 \%$ cases. Saxena et al $^{29}$ found correlation of maternal pyrexia and neonatal septicaemia in $17.34 \%$ cases. Phillips ${ }^{30}$ in their study noted maternal fever as a risk factor in $33 \%$ of cases.

In the present study, 17 [24.28\%] of cases had history of prolonged rupture of membranes [PROM] for more than 24 hours. Roy et al ${ }^{14}$ observed that $28.9 \%$ had PROM. Hossain et $\mathrm{al}^{31}$ have reported that $29.2 \%$ of septicaemia is contributed by PROM. Our finding is comparable with Roy et al ${ }^{14}$ and Hossain et al. ${ }^{31}$ Kuruvilla et $\mathrm{al}^{23}$ observed that $12.8 \%$ had PROM, which is quite lower than our observation.

\section{Distribution of Culture Positive Cases according to the Gestational Age}

Prematurity is one of the most important predisposing factors for neonatal septicaemia. In this study, those neonates who were confirmed culture positive showed distribution consistent with this. 43 [61.42\%] of the 70 positive cases have not attained their gestational maturity. However, as gestational age is not the sole determining phenomenon for this condition, neonatal septicaemia was evident in 27 [38.57\%] neonates who had completed their term before birth.

Tsering DC et al ${ }^{32}$ observed that out of 363 clinically suspected cases of septicaemia, 249 (69\%) were term and 114 (31\%) were preterm babies.

Chelliah A et al [2014] $]^{12}$ observed culture positivity in pre-terms was 14 (53.8\%).

Haseeb $\mathrm{M}$ et al ${ }^{17}$ mentioned in their study that with respect to maturity, there were 72 preterm babies $(68.57 \%)$ as compared to 33 full term babies (31.42\%).

\section{Distribution of Culture Positive Cases according to the Birth Weight}

Birth weight, just like gestational age, is another important predisposing factor for neonatal septicaemia. Newborns with low birth weight are more vulnerable to this condition. Haseeb $\mathrm{M}$ et al ${ }^{17}$ described in their study that a total of 85 cases $(80.95 \%)$ had birth weight of less than $2.5 \mathrm{~kg}$, whereas 20 cases $(19.04 \%)$ had a birth weight of $2.5 \mathrm{~kg}$ and above. When further analysed it was found that 3 cases had birth weight less than $1 \mathrm{~kg}, 23$ cases had birth weight of 1 to $1.5 \mathrm{~kg}$, 46 cases had birth weight of 1.5 to $2 \mathrm{~kg}, 13$ cases had birth weight of $2 \mathrm{~kg}$ to less than $2.5 \mathrm{~kg}$, whereas 20 cases had birth weight of $2.5 \mathrm{~kg}$ and above. Average weight of the babies with sepsis was $1.79 \mathrm{~kg}$.

\section{Distribution of Culture Positive Cases according to the Onset of Sepsis}

Jyothi $\mathrm{P}$ et $\mathrm{al}^{16}$ depicted that early-onset sepsis cases were found to be three times higher than late-onset sepsis. Out of
131 cases, 98 (74.8\%) had early-onset sepsis and 33 (25.2\%) had late-onset sepsis.

According to the study by Chelliah $\mathrm{A}$ et al ${ }^{12}$ there were $141(77.5 \%)$ cases of early-onset neonatal sepsis, out of which $86(60.99 \%)$ were culture positive. There were 41 (22.5\%) late-onset sepsis cases, out of which 24 (58.5\%) were culture positive.

The observations made in the study by Haseeb $\mathrm{M}$ et $\mathrm{al}^{17}$ was that 56 neonates (53.33\%) developed sepsis in the first 72 hours of life, i.e. early-onset sepsis, whereas 49 neonates (46.66\%) developed sepsis after 72 hours of life i.e. late-onset sepsis.

Gandhi $\mathrm{S}$ et al $^{1}$ concluded that among the 76 cases of neonatal septicaemia EOS was found in 59\% cases, whereas the rest $41 \%$ cases were of LOS.

In most of the studies except Kuruvilla et $\mathrm{al}^{5}$ study, EOS cases were more as compared to LOS. Similar to our study, Chugh et $\mathrm{al}^{33}$ had reported $68.8 \%$ cases of EOS and $32.2 \%$ cases of LOS. The observation in our study is also comparable with the study by Gandhi $S$ et al. ${ }^{1}$

\section{Bacteriology \\ Distribution of Culture Positive Cases according to Gram Reaction of the Isolate}

In our study out of 70 isolates, 35 [50\%] isolates were Grampositive cocci and 35 [50\%] isolates were Gram-negative bacilli. Kaushik et al34 [1998] mentioned isolation of Grampositive and Gram-negative bacteria equally, i.e. 50\% each. In the study by Mahapatra A et al [2002] ${ }^{15}$, of the 43 pathogens isolated 38 (88.4\%) were gram-negative bacilli and 5 (11.6\%) were gram-positive cocci. Agnihotri et al ${ }^{35}$ [2004] observed $58.5 \%$ Gram-positive and $41.5 \%$ Gram-negative organism in their study. Bhattacharjee et $\mathrm{al}^{36}$ [2008] observed that isolation of Gram-positive bacteria was $73.04 \%$ and that of Gram-negative organism was $26.96 \%$.

The observation of our study is comparable to that in the studies by Kaushik et al ${ }^{34}$ and Agnihotri et al. ${ }^{35}$

\section{Organism Wise distribution of Isolates from Positive Blood Samples}

According to the study by Jyothi $\mathrm{P}$ et al,16 aetiology of the 131 isolates included Gram-negative bacilli $(73 / 131,55.7 \%)$ and Gram-positive cocci (58/131, 44.3\%). Klebsiella spp. [30.5\%] and coagulase-negative staphylococci (CONS) [27.5\%] were the most common Gram-negative and Gram-positive organisms respectively. Roy I et al ${ }^{14}$ mentioned that of the bacterial isolates, the most frequent offender was Klebsiella spp. (24.6\%) followed by Enterobacter spp. (22.9\%), CONS (16.6\%), S. aureus (14\%), E. coli (14\%) and other less frequent isolates. Chelliah $\mathrm{A}$ et $\mathrm{al}^{12}$ concluded that most common pathogen identified was Klebsiella pneumoniae $31(28 \%)$ followed by Staphylococcus aureus 30 (27\%), Escherichia coli 14 (12.7\%), Coagulase negative Staphylococci 12 (10.9\%), Pseudomonas aeruginosa 11 (10\%), Enterococcus faecalis $8(7.2 \%)$ and Klebsiella oxytoca 4 (3.63\%). Gandhi S et al ${ }^{1}$ study observations are that E. coli [21.25\%] was the most common isolate, Staphylococcus aureus [20\%] and Klebsiella pneumoniae [20\%] were isolated in equal proportion. The next isolate was CoNS [11.25\%]. 
The observations of the present study correlate well with the study by Gandhi S et al. ${ }^{1}$

\section{Antibiotic Susceptibility Patterns}

Prior to antibiotic era, the mortality from septicaemia was $90 \%$, but it declined to $24 \%$ - $58 \%$ after antibiotics came into use. 35 The varying microbiological pattern of neonatal septicaemia warrants the need for an outgoing review of causative organisms and their antibiotic sensitivity pattern. ${ }^{14}$ The inability to be certain of infection, coupled with nonspecific signs of life threatening illness in neonates have resulted in widespread use of antibiotics, aggravating the problem of antibiotic resistance. ${ }^{37}$

\section{Staphylococci}

Roy et al14 reported that none of the gram-positive isolates were resistant to glycopeptides-vancomycin and teicoplanin. Gheibi et al ${ }^{18}$ (2008) observed that majority of gram positive isolates showed sensitivity to vancomycin and ciprofloxacin. Vancomycin is still the drug of choice for S. aureus. ${ }^{38}$

Arora et $\mathrm{al}^{39}$ noted that amongst the Gram-positive organisms, maximum resistance was seen with ampicillin $74.61 \%$ and erythromycin $69.67 \%$. An increased ampicillin resistance of $64 \%$ and $87 \%$ was also reported by Guha et al ${ }^{40}$ and Karki et al ${ }^{41}$ respectively in their studies. Mehdinejad et $\mathrm{al}^{42}$ observed that $\mathrm{S}$. aureus isolates were fully sensitive to vancomycin, amikacin and ciprofloxacin.

Penicillin-resistant S. aureus strains began emerging shortly after the introduction of penicillin in medicine in early 1940s. Today, the percentage of penicillin resistance strains has risen to $75 \%-90 \%$ with the highest rates being found among hospital strains. Similar percentages have been observed for penicillin resistant strains of S. epidermidis species group. Most penicillin resistant staphylococcal strains produce $\beta$-lactamase, which hydrolyses the $\beta$-lactam ring of antibiotic. 43

\section{Enterobacteriaceae}

Ghanshyam et al ${ }^{10}$ reported that $50 \%$ of the Klebsiella and E. coli isolates were sensitive to cefotaxime. A study by Movahedian et $\mathrm{al}^{44}$ revealed a very high degree of resistance in Gram-negative organisms, not only to commonly used antibiotics but also predominantly to broad-spectrum cephalosporins. Gheibi et al ${ }^{18}$ reported high resistance to cefotaxime $(67.5 \%)$, ceftriaxone $(65.3 \%)$ and ceftazidime (64.3\%) among Gram-negative organisms.

Ghanshyam et al ${ }^{10}$ reported that $50 \%$ of the Klebsiella and E. coli isolates were sensitive to amikacin. Mehdinejad et al ${ }^{42}$ observed that the Gram-negative bacilli showed low resistance to amikacin (28.4\%). Gheibi et al ${ }^{18}$ reported high sensitivity to amikacin $(76.5 \%)$.

Jyothi $\mathrm{P}$ et $\mathrm{al}^{16}$ observed that best overall sensitivity among Gram-negative isolates was to imipenem (93\%) followed by amikacin (52\%) and netilmicin (41\%).

Tsering DC et $\mathrm{al}^{32}$ described that most of the Gramnegative bacteria were sensitive to Ciprofloxacin except one isolate of Klebsiella. Co-trimoxazole was $100 \%$ sensitive in E. coli and Salmonella isolates, but resistant in Klebsiella. All the Citrobacter isolates were sensitive to Amikacin, Ciprofloxacin and Ofloxacin. Amikacin was effective for all the isolates of Klebsiella species, Citrobacter species and E. coli.
In the study by Roy I et al,14 most of the Gram-negative isolates of Enterobacteriaceae family were resistant to ampicillin and amoxicillin. Resistance to cefotaxime ranged from $63.7 \%$ to $65.3 \%$ and that to ceftazidime ranged from $40.8 \%$ to $53.7 \%$ of isolates. Resistance to amikacin was less frequent than resistance to gentamicin. Enterobacteria were less frequently resistant to ciprofloxacin.

Strict infection control in neonatal units, hand washing combined with judicious policy for antibiotic therapy are the main solution to this problem. It will be important, however, to continue surveillance of neonatal sepsis in order to follow closely changes in trends and risk factors, to obtain information for empiric antibiotic therapy and to react rapidly in case of major changes in susceptibility patterns and occurrence of outbreaks. ${ }^{4}$

The antimicrobial sensitivity pattern differs in different studies as well as at different times in the same hospital. 45 This is because of emergence of resistant strains as a result of indiscriminate use of antibiotics. The high resistance rates found may be associated with the frequent use of antimicrobial drugs for both prophylactic and therapeutic treatment of hospitalised newborns. ${ }^{46}$ In view of the above facts, the strategy of antibiotic usage in neonates should be reviewed periodically even in the same hospital.

\section{Limitations of the Study}

This is an observational study. As the study was restricted to neonatal age group only, sample volume for blood culture was a crucial issue. Neonates admitted in the hospital were subjected to various investigations under biochemistry, microbiology and pathology (haematology) laboratories. Sample collection generally is not that easy in neonates, mostly due to small size of blood vessels and most neonates being investigated are underweight. Multiple pricking is not advisable; hence, blood collected in single prick was distributed for three laboratories as biochemistry, microbiology and pathology (haematology). CoNS is part of normal skin flora and it may several times be isolated from blood, few times as a contaminant and as a pathogen other times. Pathogenicity of CoNS in blood should be confirmed by repeat blood culture. Retrieving a second sample for blood culture was difficult due to above reason.

The antibiotic strips used on the miniAPI system to test antibiotic susceptibility were incorporated with multiple antibiotics and all of those antibiotics were not according to CLSI guidelines of respective years of manufacturing or distribution of those strips in the market. We reported the antibiotic susceptibility to the clinicians as per the CLSI guidelines of that time, but as the antibiotics not recommended by CLSI were already tested with other recommended antibiotics the susceptibility data was recorded for analysis purpose. Same is the reason why some antibiotic combinations, which are not recommended by CLSI are mentioned in our study.

\section{CONCLUSION}

Out of total 157 samples processed, 70 blood cultures were found to be positive. Total blood culture positivity rate was $44.58 \%$.

Culture positivity was found more in male neonates as compared to females, the male-to-female ratio being 1.69: 1 . 
Highest blood culture positivity, i.e. $74.28 \%$ was found in neonates of age less than one week.

91.42\% of babies having positive blood culture were delivered in hospital setups. Out of these, $67.14 \%$ were normal vaginal births and $8.57 \%$ babies were home-based deliveries.

$17.14 \%$ out of 70 babies with positive culture were having history of maternal pyrexia during gestation. $24.28 \%$ cases were found to have prolonged rupture of membranes during deliveries. $61.42 \%$ cases were preterm deliveries. $60 \%$ babies were having low birth weight. $62.85 \%$ cases were having early-onset sepsis and $37.14 \%$ were of late-onset sepsis.

All 70 positive blood cultures were monobacterial. Among Gram-positive bacteria predominant organism was CoNS and in Gram-negative bacteria E. coli was obtained predominantly. Most common isolate in general was E. coli.

Depending on the outcome of the culture positive cases, 58 [82.85\%] cases survived.

\section{REFERENCES}

[1] Gandhi S, Ranjan KP, Ranjan N, et al. Incidence of neonatal sepsis in tertiary care hospital: an overview. Int J Med Sci Public Health 2013;2(3):548-52.

[2] Lawn JE, Cousens S, Zupan J, et al. 4 million neonatal deaths: When? Where? Why? The Lancet 2005;365(9462):891-900.

[3] Park K. Park's Textbook of preventable and social medicine. Chapter 10. 22 nd edn. Banarasidas Bhanot Publishers 2013; p. 523.

[4] Weinstein MP. Current blood culture methods and systems: clinical concepts, technology and interpretation of results. Clin Infect Dis 1996;23(1):40-6.

[5] Sankar MJ, Agarwal R, Deorari AK, et al. Sepsis in the newborn. Indian J Pediatr 2008;75(3):261-6.

[6] Package_Insert_-_93-00223_-_BacT/ALERT_PF__259794

[7] Package_Insert_-_9300222_-_BacT/ALERT_FAN__259793

[8] CLSI. Principles and procedures for blood cultures: approved guideline. Clinical and Laboratory Standards Institute document M47-A, Vol. 27 No. 17, Wayne, Pennsylvania, USA 2007.

[9] Koneman EW, Allen SD, Janda WM, et al. Colour Atlas and Textbook of diagnostic microbiology. $6^{\text {th }}$ edn. Baltimore: Lippincott Williams and Willkins 2006.

[10] Ghanshyam DK, Ramachandran VG, Gupta P. Bacteriological analysis of blood culture isolates from neonates in a tertiary care hospital in India. J Health Popul Nutr 2002;20(4):343-7.

[11] Ananthakrishnan S, Gunasekaran D. Etiology and risk factors for early onset neonatal sepsis. IJMM 2009;27(3):279.

[12] Chelliah A, Thyagarajan R, Katragadda R, et al. Isolation of MRSA, ESBL and AmpC - $\beta$-lactamases from Neonatal Sepsis at a Tertiary Care Hospital. J Clin Diagn Res 2014;8(6):DC24-DC7.

[13] Basu R, Bandyopadhyay S. Study on correlation between sepsis screening and blood culture in neonatal sepsis. IOSR Journal of Dental and Medical Sciences 2014;13(5):Ver. V. 52-6.
[14] Roy I, Jain A, Kumar M, et al. Bacteriology of neonatal septicemia in a tertiary care hospital of northern India. Indian J Med Microbiol 2002;20(3):156-9.

[15] Mahapatra A, Ghosh SK, Mishra S, et al. Enterobacter cloacae: a predominant pathogen in neonatal septicemia. Indian J Med Microbiol 2002;20(2):110-2.

[16] Jyothi P, Basavaraj MC, Basavaraj PV. Bacteriological profile of neonatal septicemia and antibiotic susceptibility pattern of the isolates. J Nat Sc Biol Med 2013;4(2):306-9.

[17] Haseeb M, Khan ST, Khan S, et al. Epidemiology clinical features and outcome of neonatal sepsis at tertiary care hospital in Sangli district of Maharashtra, INDIA: a descriptive study. Medplus - International Medical Journal 2014;1(6):351-3.

[18] Gheibi S, Fakoor Z, Karamyyar M, et al. Coagulase negative staphylococcus; the most common cause of neonatal septicemia in Urmia, Iran. Iran J Pediatr 2008;18(3):237-43.

[19] Khatua SP, Das AK, Chattergee BD, et al. Neonatal septicaemia. Indian J Pediatr 1986;53(4):509-14.

[20] Singh MB. Perinatal infections. In: Care of the newborn. $6^{\text {th }}$ edn. New Delhi: Sagar publications 2004:196-216.

[21] Stoll BJ. Infections of neonatal infant. In: Behrman RE, Kliegman RM, Jenson HB. eds. Nelson textbook of pediatrics. $17^{\text {th }}$ edn. Philadelphia: WB Saunders 2004:623-40.

[22] Patel U, Patel VK, Patel NP, et al. To evaluate C-Reactive protein and other Hematological parameters for diagnosis of Neonatal Sepsis. Int J Med Res Rev 2014;2(4):311-18.

[23] Kuruvilla KA, Pillai S, Jesudason M, et al. Bacterial profile of sepsis in a neonatal unit in south India. Indian Pediatrics 1998;35(9):851-8.

[24] Ahmed AS, Chowdhury MA, Hoque M, et al. Clinical \& Bacteriological profile of neonatal septicemia in tertiary level pediatric hospital in Bangladesh. Indian Pediatr 2002;39(11):1034-9.

[25] Knudsen FU, Steinrud J. Septicemia of the newborn, associated with ruptured fetal membranes, discolored amniotic fluid or maternal fever. Acta Pediat Scand 1976;65(6):725-31.

[26] Klein JO. Bacterial sepsis and meningitis. In: Remington JS, Klein JO. eds. Infectious diseases of the fetus and newborn infant. Philadelphia: WB Saunders 2001:943-8.

[27] Prober CG. Clinical approach to the infected neonate. In: Long SS, Pickering LK, Prober CG. eds. Principles and practice of pediatric infectious diseases. New York: Churchill Livingstone 1997:603-6.

[28] Soman M, Green B, Daling J. Risk factors for early neonatal sepsis. Am J Epidemiol 1985;121(5):712-9.

[29] Saxena S, Anand NK, Saini L, et al. Bacterial infections among home delivered neonates. Clinical picture and bacteriological profile. Indian Pediatr 1980;17(1):1724.

[30] Phillip AGS, Mills PC. Use of C-reactive protein in minimising antibiotic exposure: experience with infants initially admitted to a well-baby nursery. Pediatrics 2000;106(1):E4. 
[31] Hossain MM, Afroza S, Shirin M, et al. Bacterial etiology of neonatal sepsis in a tertiary care hospital in Bangladesh. Bang J Child Health 2004;28:81-5.

[32] Tsering DC, Chanchal L, Pal R, et al. Bacteriological profile of septicemia and the risk factors in neonates and infants in Sikkim. Journal of Global Infectious Diseases 2011;3(1):42-5.

[33] Chugh K, Aggarwal BB, Kaul VK, et al. Bacteriological profile of neonatal septicemia. Indian J Pediatr 1988;55(6):961-5.

[34] Kaushik SL, Parmar VR, Grover N, et al. Neonatal sepsis in hospital born babies. J Commun Dis 1998;30(3):147-52.

[35] Agnihotri N, Kaistha N, Gupta V. Antimicrobial susceptibility of isolates from neonatal septicemia. Jpn J Infect Dis 2004;57(6):273-5.

[36] Bhattacharjee A, Anupurba S, Gaur A, et al. Prevalence of inducible AmpC $\beta$-lactamase-producing Pseudomonas aeruginosa in a tertiary care hospital in Northen India. IJMM 2008;26(1):89-98.

[37] Jaiswal RS, Kaushal RK, Goel A, et al. Role of C-reactive protein in deciding duration of antibiotic therapy in neonatal septicemia. Indian Pediatr 2003;40(9):880-3.

[38] Jick S. Ciprofloxacin safety in a pediatric population. Pediatr Infect Dis J 1997;16(1):130-4, 160-2.

[39] Arora U, Devi P. Bacterial profile of blood stream infections and antibiotic resistance pattern of isolates. JK Sci 2007;9(4):185-90.
[40] Guha DK, Jaspal D, Das K, et al. Outcome of neonatal septicaemia - a clinical and bacteriological profile. Indian Pediatr 1978;15(5):423-7.

[41] Karki BM, Parija SC. Analysis of blood culture isolates from hospitalized neonates in Nepal. Southeast Asian J Trop Med Public Health 1999;30(3):546-8.

[42] Mehdinejad M, Khosravi AD, Morvaridi A. Study of prevalence and antimicrobial susceptibility pattern of bacteria isolated from blood cultures. J Biological Sciences 2009;9(3):249-53.

[43] Peacock SJ. Staphylococcus. In: Borriello SP, Murray PR, Funke G. eds. Topley \& Wilson's Microbiology and microbial infections. Bacteriology. 10 $0^{\text {th }}$ edn. Vol 2. Hodder Arnold Publication 2005:771-832.

[44] Movahedian AH, Moniri R, Mosayebi Z. Bacterial culture of Neonatal Sepsis. Iranian J Publ Health 2006;35(4):84-9.

[45] Mathur M, Shah H, Dixit K, et al. Bacteriological profile of neonatal septicemia cases (for the year 1990-91). J Postgrad Med 1994;40(1):18-20.

[46] Loureiro MM, de Moraes BA, Quadra MRR, et al. Study of multi-drug resistant microorganisms isolated from blood cultures of hospitalized newborns in Rio de Janeiro city, Brazil. Brazil J Microbiol 2002;33(1):73-8. 